\title{
Brain Abscess in Uncorrected Tetralogy of Fallot Diagnosed during Preparation for Computed Cardiac Angiotomography
}

Valdano Manuel ${ }^{1 *}$, Ester Matias ${ }^{2}$, Humberto Morais ${ }^{3}$, Gade Miguel ${ }^{1}$, Maria Ana Sampaio Nunes ${ }^{1}$, Adilson de Oliveira ${ }^{4}$, Jorge Manresa ${ }^{4}$ and Antonio Pedro Filipe Junior ${ }^{1}$

${ }^{1}$ Cardio-Thoracic Center, Clinica Girassol, Luanda, Angola

${ }^{2}$ Intensive Care Unit, Clinica Girassol, Luanda, Angola

${ }^{3}$ Department of Cardiology, Hospital Militar Principal / Instituto Superior, Luanda, Angola

${ }^{4}$ Neurosurgery section of Clinica Girassol, Luanda, Angola

*Corresponding author: Valdano Manuel, Centralidade do Kilamba, X41, Apt 53, Luanda, Angola, Tel: 00244917781674; E-mail: valdanympub@gmail.com

Received date: March 7, 2015; Accepted date: March 27, 2015; Published date: March 30, 2015

Copyright: () 2015 Manuel V, et al. This is an open-access article distributed under the terms of the Creative Commons Attribution License, which permits unrestricted use, distribution, and reproduction in any medium, provided the original author and source are credited.

\begin{abstract}
We describe the case of a 9-year-old boy with uncorrected tetralogy of Fallot who presented with seizures at the computed tomography room during the preparation for cardiac computed tomography angiography in order to characterize the cardiac defect. The cardiac computed tomography angiography was suspended, and a brain computed tomography without contrast was performed followed by brain magnetic resonance imaging. The brain scan showed a large abscess in the right temporo-parietal region with important midline shift. The management of the patient was a combination of antibiotic therapy and osteoplastic craniotomy with removal of abscess capsule. At the discharge the patient looks well and no focal deficits on neurological examination were observed. Now-a-days he is following up with heart surgeons for correction of the tetralogy of Fallot. The aim of this report is highlight the importance of recognizing the brain abscess in patients with uncorrected congenital heart disease. The authors also discuss the better therapeutic option of this serious often fatal complication.
\end{abstract}

Keywords: Tetralogy of fallot; Brain abscess; Congenital heart disease; Neurological complications

\section{Introduction}

Cardiovascular disease is a leading cause of mortality in all ages worldwide [1,2]. Tetralogy of Fallot (ToF) is the most common cyanotic congenital heart disease (CHD) accounting for $10 \%$. The ToF is characterized by ventricular septal defect, overriding aorta, hypertrophy of right ventricle and right ventricular outflow tract obstruction (RVOT) which can be valvular, infundibular or both [3]. There have been several reports of neurological complications associated with ToF [4,5]. Although is known, brain abscess (BA) is a serious complication in patients with uncorrected CHD mostly in the age of 4-7 years-old [4]. A PubMed search using key words "Tetralogy of Fallot" AND "Brain abscess" yield less than 10 articles in the last 10 years. We report this case to highlight the importance of recognizing the BA in patients with uncorrected CHD especially in developing countries where facilities for intervention are often unavailable. We also discuss the better therapeutic option of this serious complication.

\section{Case Report}

A 9-year-old African boy weighing $25 \mathrm{Kg}$ was referred to our cardio-thoracic center for correction of ToF, previously diagnosed at pediatric hospital David Bernardino. Past history was uneventful. In the pediatric hospital the patient presented with fever during the last five days. On admission at our center, the child presented with right temporo-parietal headache without other complaints. At physical examination cyanosis was present. The pulse was 104 per minute; blood pressure of 108/75 $\mathrm{mmHg}$ and no fever. Cardiac auscultation, revealed a grade IV/VI pansystolic murmur at the left sternal border. No focal deficit and a Glasgow of 15 points were seen on neurological examination. The remainder of the physical examination was unremarkable. Laboratory data showed hemoglobin - $13.8 \mathrm{~g} / \mathrm{dl}$, hematocrit - $42.4 \%$, leukocytes - $12.8 \times 10^{9} / \mathrm{L}$, neutrophil $71.2 \%$, Creactive protein $-2.4 \mathrm{mg} / \mathrm{dl}$. Test for plasmodium was negative. Electrocardiography showed sinus rhythm, right ventricular hypertrophy. Chest X-ray showed a "boot shaped" cardiac silhouette with an upturned apex and slight concave pulmonary artery segment (Figure 1A). No pulmonary infiltrates were found. Echocardiography showed a large VSD, nearly $40 \%$ overriding of the aorta, right ventricular hypertrophy with valvular RVOT obstruction (Figure 1B).

The patient was prepared to undergo cardiac computed tomography angiography (CCTA) according to the protocol of our cardiothoracic center for all patients with ToF. At the CCTA room the child started having seizures, and the CCTA was not done. At that moment a brain high resolution computed tomography without contrast was performed. The brain scan demonstrated an image suggestive of large abscess in the right temporo-parietal region with important midline shift (Figure 1C). There was no evidence of otitis media, paranasal sinusitis or mastoiditis. The brain magnetic resonance imaging (MRI) confirms the diagnosis of brain abscess (Figure 1D). The patient was evaluated by neurosurgeons and underwent osteoplastic craniotomy with removal of abscess capsule. Culture of the pus was positive for staphylococcus intermedium. Triple Antibiotic therapy with vancomycin, ceftriaxone and metronidazol was administered intravenously for three weeks. At the discharge the patient looks well and no focal deficits on neurological examination were observed. Now-a-days he is following up with heart surgeons for correction of the ToF. 


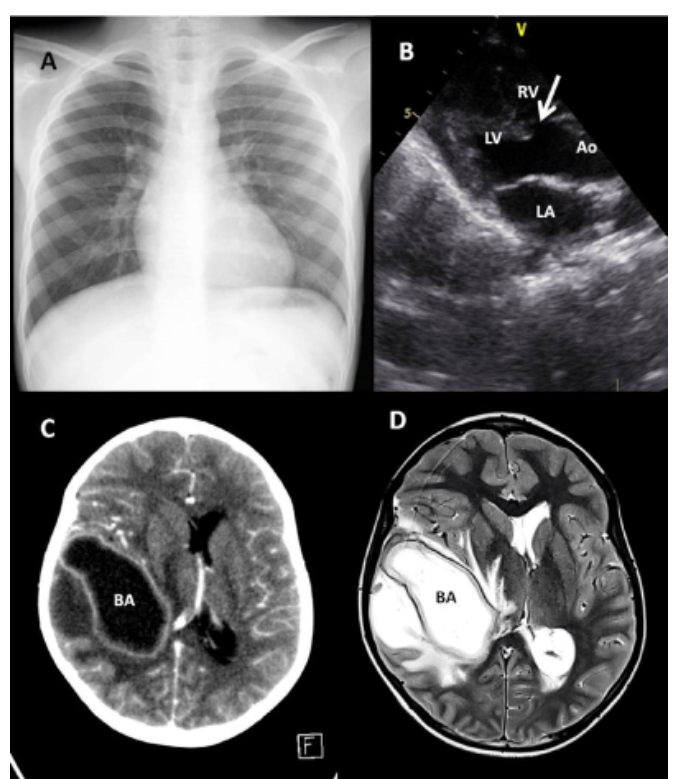

Figure 1: (A) Chest X-ray showed a "boot shaped" cardiac silhouette with an upturned apex and slight concave pulmonary artery segment. (B) Echocardiography showed a large VSD (arrow), $40 \%$ overriding of the aorta, right ventricular hypertrophy with valvular RVOT obstruction. (C) High resolution brain CT showing large an image suggestive of abscess in the right temporo-parietal region that was confirmed at brain MRI. (D) Brain MRI. Ao Aorta; BA - Brain abscess; LA - Left atrium; LV Left ventricle; RV Right ventricle

\section{Discussion}

Brain abscess is a relatively uncommon but life-threatening infection in children defined as a focal collection of infectious material within the brain. It can originate from contiguous site infections as otitis media, mastoiditis, sinusitis and rarely meningitis, after head trauma or neurosurgical procedures, from cryptogenic sources or from distant pathologic states as chronic lung infections and CHD [5,6]. The BA is a serious complication in uncorrected cyanotic CHD, although can be present in uncorrected acyanotic CHD [7]. It is largely confined to the developing countries due to delay in the diagnosis and correction due to the few pediatric cardiologists and heart surgeons as demonstrated in this case $[4,5,8]$. The peak incidence is at age $4-7$ years; our patient is 9 years-old boy.

Two factors are required for the occurrence of brain abscess: hypoxia and hyperviscosity. The first one is suggested by the fact that anaerobic bacteria are the most consistently cultured organism from the abscess. The second one is suggested by the reduced flow in the microcirculation resulting in micro or macro infarction and an infective source in shunted blood that results in focal cerebritis followed by abscess [5]. The parietal, frontal and temporal lobes are the most common sites for brain abscess [2]. In our patient the site of the abscess was right temporo-parietal.

Clinically, the patients usually present with vomiting, fever, headache, seizures, focal neurologic signs and eventually, papilloedema and coma, but these manifestations can be subtle in the initial stages $[3,5]$. In our patient although a significant abscess with midline shift was present, the initial manifestations were subtle and unspecific (fever and headache). The CT and MRI were determinant for detection and characterization of the lesion.

The management of these patients has to rely mainly on the results of retrospective studies. Optimal therapy involves a combination of high-dose parenteral antibiotics and neurosurgical drainage of the abscess $[7,9]$. This was done in our patient.

Regarding antibiotic therapy for the past 20 years high dose intravenous penicillin $\mathrm{G}$ and chloramphenicol have been used to treat brain abscess in this setting with satisfactory outcomes [9]. Actually, metronidazole is usually combined with third generation cephalosporins or penicillin $G$ for the treatment of the patients with cyanotic heart disease complicated with BA [9]. The third generation cephalosporins are much more expensive than penicillin $G$ there is a need to evaluate their effects [8]. In our case we used metronidazole and ceftriaxone.

With regards to surgery, brain abscess may be removed through osteoplastic craniotomy with removal of abscess capsule as was done in the patient presented herein, or the abscesses may be treated by aspiration and drainage of the abscess under stereotaxic guidance [4]. This procedure can be made by general or scalp block anesthesia with good result, but require expensive apparatus and expertise [3].

\section{Conclusion}

In conclusion, this case clearly shows the importance of high suspicion of brain abscess in patients with uncorrected congenital heart disease, presenting with nonspecific symptoms of fever and headache even with a normal neurological examination. The brain computerized tomography plays a crucial role in the diagnosis of this serious complication, and should be done in all patients who present in course of the disease with even a small deterioration of neurological function.

\section{References}

1. Hoffman JIE (2002) Incidence, mortality, and natural history. In: Anderson RA, Macartney F, Shinebourne EA, Tynan M, editors. Paediatric Cardiology. London: Churchill Livingstone 111-40.

2. Santulli G (2013) Epidemiology of Cardiovascular Disease in the 21st Century: Updated Numbers and Updated Facts. JCvD 1: 1-2.

3. Sethi S, Kapil S (2014) Scalp block for brain abscess drainage in a patient with uncorrected tetralogy of Fallot. WJCC 2: 934-937.

4. Kagawa M, Takeshita M, Yato S, Kitamura K (1983) Brain abscess in congenital cyanotic heart disease. J Neurosurg 58: 913-917.

5. Kumar K (2000) Neurological complications of congenital heart disease. Indian J Pediatr 67: 287-291.

6. Saez-Llorens X (2003) Brain abscess in children. Semin Pediatr Infect Dis 14: $108-114$.

7. Sung CW, Jung JH, Lee SH, Choi S, Cho JR, et al. (2010) Brain abscess in an adult with atrial septal defect. Clinical cardiology 33: E51-E53.

8. Sani MU, Mukhtar-Yola M, Karaye KM (2007) Spectrum of congenital heart disease in a tropical environment: an echocardiography study. J Natl Med Assoc 99: 665 - 669.

9. Lumbiganon P, Chaikitpinyo A (2013) Antibiotics for brain abscesses in people with cyanotic congenital heart disease. Cochrane Database Syst Rev 3: 1-13. 